Commun. Fac. Sci. Univ. Ank. Ser. A1 Math. Stat.

Volume 68, Number 2, Pages 1867-1878(2019)

DOI: $10.31801 /$ cfsuasmas.500382

ISSN 1303-5991 E-ISSN 2618-6470

http://communications.science.ankara.edu.tr/index.php?series=A1

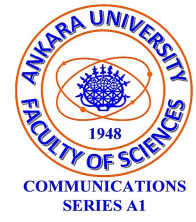

\title{
PRIME IDEALS OF NEARNESS SEMIRINGS
}

\author{
MEHMET ALI ÖZTÜRK AND IRFAN TEMUR
}

\begin{abstract}
The aim of this paper is to introduce the concept of prime (semiprime) ideals of nearness semiring theory and to introduce some properties of such ideals.
\end{abstract}

\section{INTRODUCTION}

In 1982, Pawlak introduced the concept of rough set, which is useful for modeling incompleteness and imprecision in information systems. A subset of a universe in the rough set theory, which is an extension of the set theory, is described by lower and upper approximations. An equivalence relation is a basic notion of the Pawlak rough set model. Iwinski has given an algebraic approach to rough sets 8]. Afterwards, rough subgroups were introduced by Biswas and Nanda [1. The notion of a rough ideal in a semigroup was introduced by Kuroki 9. Since then, the subject has been investigated in many papers ([2], [3], [10, [23]).

In 2002, Peters introduced near set theory, which is a generalization of rough set theory (see [17] and [18]). In this theory, Peters defined a indiscernibility relation that depends on the features of objects in order to define their nearness [21]. In his latest work, he took into consideration generalized approach theory in the work of the nearness of non-empty sets which are similar to each other [19], 20], 22].

In 2012, firstly İnan and Öztürk investigated the concept of nearness groups [5, 6] as well as and other algebraic approaches of near sets in [7], 11, [12, [13, [14], 15 .

Recently, Öztürk [16] established nearness semiring theory which is a generalization of semiring theory (see [4]) and analyzed some properties of nearness semirings and ideals.

In this paper, the concept of prime (semiprime) ideals of nearness semiring theory is introduced and some properties of such ideals are given.

Received by the editors: December 21, 2018; Accepted: January 28, 2019.

2010 Mathematics Subject Classification. Primary 05C38, 15A15; Secondary 05A15, 15A18.

Key words and phrases. Semigroups, semirings, near sets, weak nearness approximation spaces, nearness semirings.

(C)2019 Ankara University Communications Faculty of Sciences University of Ankara-Series A1 Mathematics and Statistics 


\section{Preliminaries}

For an object $x \in X$, an object description is specified via a tuple of function values $\Phi(x)$. Assume that $B \subseteq \mathcal{F}$ is a set of functions representing properties of sample objects $X \subseteq \mathcal{O}$. Take $\varphi_{i} \in B$, where $\varphi_{i}: \mathcal{O} \rightarrow \mathbb{R}$. The functions standing for object properties supply a basis for an object description $\Phi: \mathcal{O} \rightarrow \mathbb{R}^{L}$, $\Phi(x)=\left(\varphi_{1}(x), \varphi_{2}(x), \ldots, \varphi_{L}(x)\right)$ a vector holding measurements (returned values) linked with each functional value $\varphi_{i}(x)$, with description length $|\Phi|=L$, where $L$ is a positive integer ([19]).

Sample objects $X \subseteq \mathcal{O}$ are near each other if and only if the objects have similar descriptions. The selection of functions $\varphi_{i} \in B$ used to specify an object of interest is very significant to consider. Recall that each $\varphi$ provides a description of an object. So, let $\triangle_{\varphi_{i}}$ denote $\triangle_{\varphi_{i}}=\left|\varphi_{i}\left(x^{\prime}\right)-\varphi_{i}(x)\right|$, where $x^{\prime}, x \in \mathcal{O}$. Peters investigated the difference $\varphi$ that leads to a description of the indiscernibility relation " $\sim_{B}$ " [19].

Definition 1. ([19]) Let $x, x^{\prime} \in \mathcal{O}, B \subseteq \mathcal{F}$.

$$
\sim_{B}=\left\{(x, x) \in \mathcal{O} \times \mathcal{O} \mid \triangle_{\varphi_{i}}=0 \text { for all } \varphi_{i} \in B\right\}
$$

is called the indiscernibility relation on $\mathcal{O}$, where description length $i \leq|\Phi|$.

The basic idea in the near set approach to object recognition is to compare object descriptions. Sets of objects $X, X^{\prime}$ are considered near each other, if the sets contain objects with at least partial matching descriptions.

Definition 2. ([19]) Let $X, X^{\prime} \subseteq \mathcal{O}, B \subseteq \mathcal{F}$. Set $X$ is called near $X^{\prime}$ if there exist $x \in X, x^{\prime} \in X^{\prime}, \varphi_{i} \in B$ such that $x \sim_{\varphi_{i}} x^{\prime}$.

\begin{tabular}{l|l} 
Symbol & Interpretation \\
\hline$B$ & $B \subseteq \mathcal{F}$, set of probe functions, \\
$r$ & $\left(\begin{array}{c}|B| \\
r\end{array}\right)$, i.e.,$|B|$ probe functions $\varphi_{i} \in B$ taken $r$ at a time, \\
$B_{r}$ & $r \leq|B|$ probe functions in $B$, \\
$\sim_{B_{r}}$ & indiscernibility relation defined using $B_{r}$, \\
{$[x]_{B_{r}}$} & {$[x]_{B_{r}}=\left\{x^{\prime} \in \mathcal{O} \mid x \sim_{B_{r}} x^{\prime}\right\}$, near equivalence class, } \\
$\mathcal{O} / \sim_{B_{r}}$ & $\mathcal{O} / \sim_{B_{r}}=\left\{[x]_{B_{r}} \mid x \in \mathcal{O}\right\}=\xi_{\mathcal{O}, B_{r}, \text { quotient set, }}$, \\
$N_{r}(B)$ & $N_{r}(B)=\left\{\xi \mathcal{O}, B_{r} \mid B_{r} \subseteq B\right\}$, set of partitions, \\
$\nu_{N_{r}}$ & $\nu_{N_{r}}: \wp(\mathcal{O}) \times \wp(\mathcal{O}) \rightarrow[0,1]$, overlap function, \\
$N_{r}(B)_{*} X$ & $N_{r}(B)_{*} X=\bigcup_{[x]_{B_{r}} \subseteq X}^{[x]_{B_{r}}, \text { lower approximation, }}$ \\
$N_{r}(B)^{*} X$ & $N_{r}(B)^{*} X=\bigcup_{[x]_{B_{r}} \cap X \neq \varnothing},[x]_{B_{r}}$, upper approximation, \\
$B n d_{N_{r}(B)}(X)$ & $N_{r}(B)^{*} X \backslash N_{r}(B)_{*} X=\left\{x \in N_{r}(B)^{*} X \mid x \notin N_{r}(B)_{*} X\right\}$.
\end{tabular}

Table 1 : Symbols of Nearness Approximation Space 
A nearness approximation space is a tuple $\left(\mathcal{O}, \mathcal{F}, \sim_{B_{r}}, N_{r}, \nu_{N_{r}}\right)$ where the approximation space is defined with a set of perceived objects $O$, set of probe functions $\mathcal{F}$ representing object features, $\sim_{B_{r}}$ indiscernibility relation $B_{r}$ defined relative to $B_{r} \subseteq B \subseteq \mathcal{F}$, collection of partitions (families of neighborhoods) $N_{r}(B)$, and overlap function $\nu_{N_{r}}([19])$.

In [15, since $\nu_{N_{r}}: \wp(\mathcal{O}) \times \wp(\mathcal{O}) \rightarrow[0,1]$ is not needed, which is overlap function, is not needed when algebraic structures are studied on the nearness approximation space $\left(\mathcal{O}, \mathcal{F}, \sim_{B_{r}}, N_{r}, \nu_{N_{r}}\right)$, the following definition was given.

Definition 3. (15]) Let $\mathcal{O}$ be a set of perceived objects, $\mathcal{F}$ be a set of the probe functions, $\sim_{B_{r}}$ be an indiscernibility relation, and $N_{r}(B)$ be a collection of partitions. Then, $\left(\mathcal{O}, \mathcal{F}, \sim_{B_{r}}, N_{r}\right)$ is called a weak nearness approximation space.

Theorem 1. (15) Let $\left(\mathcal{O}, \mathcal{F}, \sim_{B_{r}}, N_{r}\right)$ be a weak nearness approximation space and $X, Y \subset \mathcal{O}$, then the following statements hold;

i) $N_{r}(B)_{*} X \subseteq X \subseteq N_{r}(B)^{*} X$,

ii) $N_{r}(B)^{*}(X \cup Y)=\left(N_{r}(B)^{*} X\right) \cup\left(N_{r}(B)^{*} Y\right)$,

iii) $N_{r}(B)_{*}(X \cap Y)=\left(N_{r}(B)_{*} X\right) \cap\left(N_{r}(B)_{*} Y\right)$,

iv) $X \subseteq Y$ implies $N_{r}(B)_{*} X \subseteq N_{r}(B)_{*} Y$,

v) $X \subseteq Y$ implies $N_{r}(B)^{*} X \subseteq N_{r}(B)^{*} Y$,

vi) $N_{r}(B)_{*}(X \cup Y) \supseteq\left(N_{r}(B)_{*} X\right) \cup\left(N_{r}(B)_{*} Y\right)$,

vii) $N_{r}(B)^{*}(X \cap Y) \subseteq\left(N_{r}(B)^{*} X\right) \cap\left(N_{r}(B)^{*} Y\right)$.

Definition 4. ([16]) Let $\left(\mathcal{O}, \mathcal{F}, \sim_{B_{r}}, N_{r}\right)$ be a weak nearness approximation space and $S \subset \mathcal{O}$. $S$ is called a semiring on $\mathcal{O}$ if the following properties are satisfied:

$\left.N S R_{1}\right)(S,+)$ is an abelian monoid on $\mathcal{O}$ with identity element 0 ,

$\left.N S R_{2}\right)(S, \cdot)$ is a monoid on $\mathcal{O}$ with identity element 1 ,

$\left.N S R_{3}\right)$ For all $x, y, z \in S$,

$$
x \cdot(y+z)=(x \cdot y)+(x \cdot z) \text { and }(x+y) \cdot z=(x \cdot z)+(y \cdot z)
$$

hold in $N_{r}(B)^{*} S$,

$\left.N S R_{4}\right)$ For all $x \in S$,

$$
0 \cdot x=0=x \cdot 0
$$

hold in $N_{r}(B)^{*} S$,

$\left.N S R_{5}\right) 1 \neq 0$.

Lemma 1. ([16]) Let $(S,+, \cdot)$ be a nearness semiring. If $\sim_{B_{r}}$ is a congruence indiscernibility relation on $S$, then $[x]_{B_{r}}+[y]_{B_{r}} \subseteq[x+y]_{B_{r}}$ and $[x]_{B_{r}} \cdot[y]_{B_{r}} \subseteq[x \cdot y]_{B_{r}}$ for all $x, y \in S$.

Definition 5. ([16] $)$ Let $(S,+, \cdot)$ be a nearness semiring, $B_{r} \subseteq \mathcal{F}$, where $r \leq|B|$ and $B \subseteq \mathcal{F}, \sim_{B_{r}}$ be an indiscernibility relation on weak nearness approximation space $\mathcal{O}$. Then, $\sim_{B_{r}}$ is called a complete congruence indiscernibility relation on nearness semiring $S$ if $[x]_{B_{r}}+[y]_{B_{r}}=[x+y]_{B_{r}}$ and $[x]_{B_{r}} \cdot[y]_{B_{r}}=[x \cdot y]_{B_{r}}$ for all $x, y \in S$. 
Let $(S,+, \cdot)$ be a nearness semiring. Let $X+Y=\{x+y \mid x \in X$ and $y \in Y\}$ and $X \cdot Y=\left\{\sum_{\text {finite }} x_{i} \cdot y_{i} \mid x_{i} \in X\right.$ and $\left.y_{i} \in Y\right\}$, where subsets $X$ and $Y$ of $S$.

Definition 6. ([16]) Let $(S,+, \cdot)$ be a nearness semiring, and $A$ be a subsemiring of $S$, where $A \neq S$.

i) $A$ is called a right (left) ideals of $S$ if $A \cdot S \subseteq N_{r}(B)^{*} A\left(S \cdot A \subseteq N_{r}(B)^{*} A\right)$.

ii) $A$ is called an upper-near right (left) ideals of $S$ if $\left(N_{r}(B)^{*} A\right) \cdot S \subseteq N_{r}(B)^{*} A$ $\left(S \cdot\left(N_{r}(B)^{*} A\right) \subseteq N_{r}(B)^{*} A\right)$.

Theorem 2. ([16]) Let $(S,+, \cdot)$ be a nearness semiring. The following properties hold:

i) if $\varnothing \neq A \subseteq S, A+A \subseteq A$ and $A \cdot A \subseteq A$, then $A$ is an upper-near right (left) ideal of $S$.

ii) if $A$ is a right (left) ideal of $S$, and $N_{r}(B)^{*}\left(N_{r}(B)^{*} A\right)=N_{r}(B)^{*} A$, then $A$ is an upper-near right (left) ideal of $S$.

Theorem 3. ([16]) Let $(S,+, \cdot)$ be a nearness semiring, $\left\{A_{i} \mid i \in I\right\}$ be a set of ideals of $S$, where an arbitrary index set $I$.

i) If $N_{r}(B)^{*}\left(\bigcap_{i \in I} A_{i}\right)=\bigcap_{i \in I} N_{r}(B)^{*} A_{i}$, then $\bigcap_{i \in I} A_{i}$ is a ideal of $S$.

ii) $\bigcup_{i \in I} A_{i}$ is a ideal of $S$.

For other notions and definitions not mentioned in this paper, the readers are referred to [4], 19], 20], [7, [15], and [16].

\section{Prime Ideals of Nearness Semirings}

Definition 7. Let $S$ be a nearness semiring and $P$ be an ideal of $S . P$ is called a prime (resp. semiprime) ideal of $S$ if $A_{1} \cdot A_{2} \subseteq N_{r}(B)^{*} P\left(A^{2}=A \cdot A \subseteq N_{r}(B)^{*} P\right)$ implies $A_{1} \subseteq P$ or $A_{2} \subseteq P$ (resp. $A \subseteq P$ ) for any ideals $A_{1}$ and $A_{2}$ of $S$ (resp. for any ideal $A$ of $S$ ).

Definition 8. Let $S$ be a nearness semiring and $P$ be an ideal of $S . P$ is called an upper-near prime ( resp. semiprime) ideal of $S$ if $\left(N_{r}(B)^{*} A_{1}\right) \cdot\left(N_{r}(B)^{*} A_{2}\right) \subseteq$ $N_{r}(B)^{*} P\left(\right.$ resp. $\left.\left(N_{r}(B)^{*} A\right) \cdot\left(N_{r}(B)^{*} A\right) \subseteq N_{r}(B)^{*} P\right)$ implies $N_{r}(B)^{*} A_{1} \subseteq P$ or $N_{r}(B)^{*} A_{2} \subseteq P\left(\right.$ resp. $\left.N_{r}(B)^{*} A \subseteq P\right)$ for any ideals $A_{1}$ and $A_{2}$ of $S$ (resp. for any ideal $A$ of $S)$.

Theorem 4. Let $S$ be a nearness semiring, $A_{1}, A_{2}$ and $P$ are ideals of $S$ such that $N_{r}(B)^{*}\left(N_{r}(B)^{*} A_{1}\right)=N_{r}(B)^{*} A_{1}, N_{r}(B)^{*}\left(N_{r}(B)^{*} A_{2}\right)=N_{r}(B)^{*} A_{2}$ and

$N_{r}(B)^{*}\left(N_{r}(B)^{*} P\right)=N_{r}(B)^{*} P$, respectively. If $P$ is a prime ideals and $\left(N_{r}(B)^{*} A_{1}\right)$. $\left(N_{r}(B)^{*} A_{2}\right) \subseteq N_{r}(B)^{*} P$, then $P$ is an upper-near prime ideal of $S$.

Proof. Since $P$ is a prime ideal of $S$ such that $N_{r}(B)^{*}\left(N_{r}(B)^{*} P\right)=N_{r}(B)^{*} P$, $P$ is an upper-near ideal of $S$ by Theorem 2(ii). Suppose that $\left(N_{r}(B)^{*} A_{1}\right)$. 
$\left(N_{r}(B)^{*} A_{2}\right) \subseteq N_{r}(B)^{*} P$ such that $N_{r}(B)^{*} A_{1} \nsubseteq P$ or $N_{r}(B)^{*} A_{2} \nsubseteq P$. Then, there exists an element $x \in N_{r}(B)^{*} A_{1}$ such that $x \notin P$ and $y \in N_{r}(B)^{*} A_{2}$ such that $y \notin P$. From here, $[x]_{B_{r}} \cap A \neq \varnothing$ and $[y]_{B_{r}} \cap B \neq \varnothing \Rightarrow a_{1} \in[x]_{B_{r}}, a_{1} \in A_{1}$ and $a_{2} \in[x]_{B_{r}}, a_{2} \in A_{2} \Rightarrow x \sim_{B_{r}} a_{1}, a_{2} \in A_{1}$ and $y \sim_{B_{r}} a_{2}, a_{2} \in A_{2}$. Since $\sim_{B_{r}}$ is a congruence indiscernibility relation on $S$, and $A_{1}$ and $A_{2}$ are ideals of $S$, we have $x y \sim_{B_{r}} a_{1} a_{2}, a_{1} a_{2} \in\left(N_{r}(B)^{*} A_{1}\right) \cap\left(N_{r}(B)^{*} A_{2}\right)$. Thus, $[x y]_{B_{r}} \cap$ $\left(\left(N_{r}(B)^{*} A_{1}\right) \cap\left(N_{r}(B)^{*} A_{2}\right)\right) \neq \varnothing \Rightarrow x y \in N_{r}(B)^{*}\left(\left(N_{r}(B)^{*} A_{1}\right) \cap\left(N_{r}(B)^{*} A_{2}\right)\right) \Rightarrow$ $x y \in N_{r}(B)^{*}\left(\left(N_{r}(B)^{*} A_{1}\right)\right.$ and $\left.x y \in N_{r}(B)^{*}\left(N_{r}(B)^{*} A_{2}\right)\right)$ by Theorem1.(vii). From hypothesis, we get $x y \in N_{r}(B)^{*} A_{1}$ and $x y \in N_{r}(B)^{*} A_{2} \Rightarrow(x y)^{2}=(x y)(x y) \in$ $\left(N_{r}(B)^{*} A_{1}\right) \cdot\left(N_{r}(B)^{*} A_{2}\right)$, and so $(x y)^{2} \in N_{r}(B)^{*} P$. Since $P$ is a prime ideal, $x y \in P$ which is a contradiction. Hence, either $N_{r}(B)^{*} A_{1} \subseteq P$ or $N_{r}(B)^{*} A_{2} \subseteq P$.

We give the following theorem without the proof that is similar to the above proof.

Theorem 5. Let $S$ be a nearness semiring, $A$ and $P$ are ideals of $S$ such that $N_{r}(B)^{*}\left(N_{r}(B)^{*} A\right)=N_{r}(B)^{*} A$ and $N_{r}(B)^{*}\left(N_{r}(B)^{*} P\right)=N_{r}(B)^{*} P$, respectively. If $P$ is a semiprime ideals and $\left(N_{r}(B)^{*} A\right) \cdot\left(N_{r}(B)^{*} A\right) \subseteq N_{r}(B)^{*} P$, then $P$ is an upper-near semiprime ideal of $S$.

Let $A$ be a non-empty subset of nearness semiring $S$ and $s \in S$. Let $s \cdot A=$ $\left\{\sum_{\text {finite }} s a_{i} \mid a_{i} \in A\right\}$.

Lemma 2. Let $S$ be a nearness semiring. Then $a \cdot S$ is a right ideal of $S$ for any $a \in S$.

Proof. Let $x, y \in a \cdot S$. In this case, $x=\sum_{i=1} a s_{i} ; s_{i} \in S$ and $y=\sum_{i=1} a s_{i}^{\prime} ; s_{i}^{\prime} \in S$. Thus, $x+y=\sum_{i=1} a s_{i}+\sum_{i=1} a s_{i}^{\prime}=\sum_{i=1} a \hat{s}_{i}=a \sum_{i=1} \hat{s}_{i} \in a \cdot\left(N_{r}(B)^{*} S\right)$ for all $\hat{s} \in S$. There exists $z \in N_{r}(B)^{*} S$ such that $x+y=a z$ for any $a \in S, z \in N_{r}(B)^{*} S$. Then $[z]_{B_{r}} \cap S \neq \varnothing \Rightarrow c \in[z]_{B_{r}}, c \in S \Rightarrow z \sim_{B_{r}} c, c \in S$.Since $\sim_{B_{r}}$ is a congruence indiscernibility relation on $S$, we get $a z \sim_{B_{r}} a c, c \in S \Rightarrow a c \in[a z]_{B_{r}}$ and $a c \in a \cdot S$ $\Rightarrow[a z]_{B_{r}} \cap(a \cdot S) \neq \varnothing$, so we obtain $x+y=a z \in N_{r}(B)^{*}(a \cdot S)$, namely, $a \cdot S+a \cdot S$ $\subseteq N_{r}(B)^{*}(a \cdot S)$.

Now let $x \in a \cdot S, s \in S$. Thus, $x=\sum_{i=1} a s_{i} ; s_{i} \in S$. Therefore, $x s=\left(\sum_{i=1} a s_{i}\right) s=$ $\sum_{i=1}\left(a s_{i}\right) s=\sum_{i=1} a\left(s_{i} s\right)=a \sum_{i=1} s_{i} s \in a \cdot\left(N_{r}(B)^{*} S\right)$, and so there exists $c \in N_{r}(B)^{*} S$ such that $x s=a c$ for all $s \in S, c \in N_{r}(B)^{*} S$. Therefore $[c]_{B_{r}} \cap S \neq \varnothing \Rightarrow$ $z \in[c]_{B_{r}}, z \in S \Rightarrow c \sim_{B_{r}} z, z \in S$. Since $\sim_{B_{r}}$ is a congruence indiscernibility relation on $S$, we get that $a c \sim_{B_{r}} a z, z \in S \Rightarrow a z \in[a c]_{B_{r}}$ and $a z \in a \cdot S$ $\Rightarrow[a c]_{B_{r}} \cap(a \cdot S) \neq \varnothing$, so we have $x s=a c \in N_{r}(B)^{*}(a \cdot S)$. Thus, we obtain $(a \cdot S) \cdot S \subseteq N_{r}(B)^{*}(a \cdot S)$.

In general, the intersection of ideals of the nearness semiring $S$ is not an ideal, as shown in the following. 
Example 1. Let $\mathcal{O}=\{0,1, a, b, c, d, e, f, g, h, i, j, k, n\}$ be a set of perceptual objects where

$$
\begin{aligned}
& 0=\left[\begin{array}{ll}
0 & 0 \\
0 & 0
\end{array}\right], 1=\left[\begin{array}{ll}
1 & 0 \\
0 & 1
\end{array}\right], a=\left[\begin{array}{ll}
1 & 0 \\
0 & 0
\end{array}\right], b=\left[\begin{array}{ll}
0 & 0 \\
1 & 0
\end{array}\right], \\
& c=\left[\begin{array}{ll}
0 & 0 \\
0 & 1
\end{array}\right], d=\left[\begin{array}{ll}
0 & 1 \\
0 & 0
\end{array}\right], e=\left[\begin{array}{ll}
1 & 0 \\
1 & 0
\end{array}\right], f=\left[\begin{array}{ll}
0 & 0 \\
1 & 1
\end{array}\right], \\
& g=\left[\begin{array}{ll}
0 & 1 \\
0 & 1
\end{array}\right], h=\left[\begin{array}{ll}
1 & 1 \\
0 & 0
\end{array}\right], i=\left[\begin{array}{ll}
0 & 1 \\
1 & 0
\end{array}\right], j=\left[\begin{array}{ll}
1 & 1 \\
1 & 0
\end{array}\right], \\
& k=\left[\begin{array}{ll}
1 & 0 \\
1 & 1
\end{array}\right], l=\left[\begin{array}{ll}
0 & 1 \\
1 & 1
\end{array}\right], m=\left[\begin{array}{ll}
1 & 1 \\
0 & 1
\end{array}\right], n=\left[\begin{array}{ll}
1 & 1 \\
1 & 1
\end{array}\right]
\end{aligned}
$$

for $U=\left\{\left[a_{i j}\right]_{2 x 2} \mid a_{i j} \in \mathbb{Z}_{2}\right\}, r=1, B=\left\{\varphi_{1}, \varphi_{2}, \varphi_{3}\right\} \subseteq \mathcal{F}$ be a set of probe functions, $S=\{a, b, d, e, h\} \subset \mathcal{O}, A_{1}=\{a, h\} \subseteq S$ and $A_{2}\{b, h\} \subseteq S$. Values of the probe functions

$$
\begin{aligned}
& \varphi_{1}: \mathcal{O} \rightarrow V_{1}=\left\{\alpha_{1}, \alpha_{2}, \alpha_{3}, \alpha_{4}, \alpha_{5}\right\}, \\
& \varphi_{2}: \mathcal{O} \rightarrow V_{2}=\left\{\alpha_{1}, \alpha_{3}, \alpha_{4}, \alpha_{6}\right\}, \\
& \varphi_{3}: \mathcal{O} \rightarrow V_{3}=\left\{\alpha_{1}, \alpha_{3}, \alpha_{4}, \alpha_{5}, \alpha_{6}\right\}
\end{aligned}
$$

are given in Table 2 .

\begin{tabular}{c|cccccccccccccc} 
& 0 & 1 & $a$ & $b$ & $c$ & $d$ & $e$ & $f$ & $g$ & $h$ & $i$ & $j$ & $k$ & $n$ \\
\hline$\varphi_{1}$ & $\alpha_{1}$ & $\alpha_{2}$ & $\alpha_{1}$ & $\alpha_{3}$ & $\alpha_{1}$ & $\alpha_{3}$ & $\alpha_{4}$ & $\alpha_{3}$ & $\alpha_{4}$ & $\alpha_{3}$ & $\alpha_{1}$ & $\alpha_{4}$ & $\alpha_{5}$ & $\alpha_{5}$ \\
$\varphi_{2}$ & $\alpha_{3}$ & $\alpha_{3}$ & $\alpha_{4}$ & $\alpha_{3}$ & $\alpha_{1}$ & $\alpha_{1}$ & $\alpha_{4}$ & $\alpha_{3}$ & $\alpha_{4}$ & $\alpha_{4}$ & $\alpha_{3}$ & $\alpha_{4}$ & $\alpha_{6}$ & $\alpha_{6}$ \\
$\varphi_{3}$ & $\alpha_{3}$ & $\alpha_{3}$ & $\alpha_{1}$ & $\alpha_{1}$ & $\alpha_{4}$ & $\alpha_{4}$ & $\alpha_{6}$ & $\alpha_{1}$ & $\alpha_{3}$ & $\alpha_{6}$ & $\alpha_{3}$ & $\alpha_{6}$ & $\alpha_{5}$ & $\alpha_{6}$
\end{tabular}

Table 2

Let us now determine the near equivalence classes according to the indiscernibility relation $\sim_{B_{r}}$ of elements in $\mathcal{O}:$

$$
\begin{aligned}
{[0]_{\varphi_{1}} } & =\left\{x \in \mathcal{O} \mid \varphi_{1}(x)=\varphi_{1}(0)=\alpha_{1}\right\}=\{0, a, c, i\} \\
& =[a]_{\varphi_{1}}=[c]_{\varphi_{1}}=[i]_{\varphi_{1}}, \\
{[1]_{\varphi_{1}} } & =\left\{x \in \mathcal{O} \mid \varphi_{1}(x)=\varphi_{1}(1)=\alpha_{2}\right\}=\{1\}, \\
{[b]_{\varphi_{1}} } & =\left\{x \in \mathcal{O} \mid \varphi_{1}(x)=\varphi_{1}(a)=\alpha_{3}\right\}=\{b, d, f, h\} \\
& =[d]_{\varphi_{1}}=[f]_{\varphi_{1}}=[h]_{\varphi_{1}}, \\
{[e]_{\varphi_{1}} } & =\left\{x \in \mathcal{O} \mid \varphi_{1}(x)=\varphi_{1}(e)=\alpha_{4}\right\}=\{e, g, j\}, \\
& =[g]_{\varphi_{1}}=[j]_{\varphi_{1}}, \\
{[k]_{\varphi_{1}} } & =\left\{x \in \mathcal{O} \mid \varphi_{1}(x)=\varphi_{1}(e)=\alpha_{5}\right\}=\{k, n\} \\
& =[n]_{\varphi_{1}} .
\end{aligned}
$$


Then, we get $\xi_{\varphi_{1}}=\left\{[0]_{\varphi_{1}},[1]_{\varphi_{1}},[b]_{\varphi_{1}},[e]_{\varphi_{1}}[k]_{\varphi_{1}}\right\}$.

$$
\begin{aligned}
{[0]_{\varphi_{2}} } & =\left\{x \in \mathcal{O} \mid \varphi_{2}(x)=\varphi_{2}(0)=\alpha_{3}\right\}=\{0,1, b, f, i\} \\
& =[1]_{\varphi_{2}}=[b]_{\varphi_{2}}=[f]_{\varphi_{2}}=[i]_{\varphi_{2}}, \\
{[a]_{\varphi_{2}} } & =\left\{x \in \mathcal{O} \mid \varphi_{2}(x)=\varphi_{2}(a)=\alpha_{4}\right\}=\{a, e, g, h, j\} \\
& =[e]_{\varphi_{2}}=[g]_{\varphi_{2}}=[h]_{\varphi_{2}}=[j]_{\varphi_{2}}, \\
{[c]_{\varphi_{2}} } & =\left\{x \in \mathcal{O} \mid \varphi_{2}(x)=\varphi_{2}(\gamma)=\alpha_{1}\right\}=\{c, d\} \\
& =[d]_{\varphi_{2}}, \\
{[k]_{\varphi_{2}} } & =\left\{x \in \mathcal{O} \mid \varphi_{2}(x)=\varphi_{2}(k)=\alpha_{6}\right\}=\{k, n\}, \\
& =[n]_{\varphi_{2}} .
\end{aligned}
$$

Thus, we have $\xi_{\varphi_{2}}=\left\{[0]_{\varphi_{2}},[a]_{\varphi_{2}},[c]_{\varphi_{2}}[k]_{\varphi_{2}}\right\}$.

$$
\begin{aligned}
{[0]_{\varphi_{3}} } & =\left\{x \in \mathcal{O} \mid \varphi_{3}(x)=\varphi_{3}(0)=\alpha_{3}\right\}=\{0,1, g, i\} \\
& =[1]_{\varphi_{3}}=[g]_{\varphi_{3}}=[i]_{\varphi_{3}}, \\
{[a]_{\varphi_{3}} } & =\left\{x \in \mathcal{O} \mid \varphi_{3}(x)=\varphi_{3}(a)=\alpha_{1}\right\}=\{a, b, f\} \\
& =[b]_{\varphi_{3}}=[f]_{\varphi_{3}}, \\
{[c]_{\varphi_{3}} } & =\left\{x \in \mathcal{O} \mid \varphi_{3}(x)=\varphi_{3}(c)=\alpha_{4}\right\}=\{c, d\} \\
& =[d]_{\varphi_{3}}, \\
{[e]_{\varphi_{3}} } & =\left\{x \in \mathcal{O} \mid \varphi_{3}(x)=\varphi_{3}(e)=\alpha_{6}\right\}=\{e, h, j, n\} \\
& =[h]_{\varphi_{2}}=[j]_{\varphi_{3}}=[n]_{\varphi_{3}} \\
{[k]_{\varphi_{3}} } & =\left\{x \in \mathcal{O} \mid \varphi_{3}(x)=\varphi_{3}(k)=\alpha_{5}\right\}=\{k\} .
\end{aligned}
$$

Hence, we obtain $\xi_{\varphi_{3}}=\left\{[0]_{\varphi_{3}},[a]_{\varphi_{3}},[c]_{\varphi_{3}},[e]_{\varphi_{3}},[k]_{\varphi_{3}}\right\}$. Therefore, for $r=1, a$ set of partitions of $\mathcal{O}$ is $N_{r}(B)=\left\{\xi_{\varphi_{1}}, \xi_{\varphi_{2}}, \xi_{\varphi_{3}}\right\}$. Then we can write

$$
\begin{aligned}
N_{1}(B)^{*} S & =\bigcup_{\left.[x]_{\varphi_{i}} \cap S \neq \varnothing\right]_{\varphi_{i}}} \cup[a]_{\varphi_{1}} \cup[e]_{\varphi_{1}} \cup[a]_{\varphi_{2}} \cup[c]_{\varphi_{2}} \cup[a]_{\varphi_{3}} \cup[c]_{\varphi_{3}} \cup[e]_{\varphi_{3}} \\
& =[0]_{\varphi_{1}} \cup[a, i, j\} \\
& =\{0, a, b, c, d, e, f, g, h, i, j,
\end{aligned}
$$


Considering the following table of operation:

\begin{tabular}{c|ccccc}
+ & $a$ & $b$ & $d$ & $e$ & $h$ \\
\hline$a$ & 0 & $e$ & $h$ & $b$ & $d$ \\
$b$ & $e$ & 0 & $i$ & $b$ & $f$ \\
$d$ & $h$ & $i$ & 0 & $j$ & $a$ \\
$e$ & $b$ & $a$ & $j$ & 0 & $i$ \\
$h$ & $d$ & $j$ & $a$ & $i$ & 0
\end{tabular}

In that case, $(S,+)$ is an abelian monoid on $\mathcal{O}$ with identity element 0. Considering the following table of operation:

\begin{tabular}{c|ccccc}
$\cdot$ & $a$ & $b$ & $d$ & $e$ & $h$ \\
\hline$a$ & $a$ & 0 & $d$ & $a$ & $h$ \\
$b$ & $b$ & 0 & $c$ & 0 & $d$ \\
$d$ & 0 & $a$ & 0 & $a$ & 0 \\
$e$ & $e$ & 0 & $g$ & $e$ & $n$ \\
$h$ & $a$ & $e$ & 0 & $e$ & 0
\end{tabular}

Then $(S, \cdot)$ is a semigroup on $\mathcal{O}$. Also, $(S,+, \cdot)$ satisfies conditions $\left(N S R_{3}\right)$, $\left(N S R_{4}\right)$ and $\left(N S R_{5}\right)$. Therefore, $(S,+, \cdot)$ is a semiring on the weak nearness approximation space $\mathcal{O}$ by Definition 4 , i.e. , $(S,+, \cdot)$ is a $\Gamma$-nearness semiring. Moreover,

$$
\begin{aligned}
N_{1}(B)^{*} A_{1} & =\bigcup_{\left.[x]_{\varphi_{i}} \cap{ }^{\cap}\right]_{\varphi_{i}} \neq \varnothing} \\
& =\{0, a, b, c, d, f, g, h, i, j, n\} .
\end{aligned}
$$

Considering the above table of operations, $A_{1}$ is an ideal of $S$.

$$
\begin{aligned}
N_{1}(B)^{*} A_{2} & =\bigcup_{[x]_{\varphi_{i}} \cap[x]_{\varphi_{i}}} \cap A_{2} \neq \varnothing \\
& =\{0, a, b, c, d, e, f, g, h, i, j\} .
\end{aligned}
$$

Similarly, $A_{2}$ is an ideal of $S$. Also,

$$
\begin{aligned}
N_{1}(B)^{*}\left(A_{1} \cap A_{2}\right) & =\bigcup_{[x]_{\varphi_{i}} \cap\left(A_{1} \cap A_{\varphi_{2}}\right) \neq \varnothing} \\
& =\{a, b, d, e, f, g, h, j, n\} .
\end{aligned}
$$

In this case, let $h \in A_{1} \cap A_{2}=\{h\}$, and so $h+h=0 \notin N_{1}(B)^{*}\left(A_{1} \cap A_{2}\right)$. Therefore, $A_{1} \cap A_{2}$ is not an ideal of $S$ by Definition 6 . Furthermore, considering 
the following tables of operations:

\begin{tabular}{c|cccccccccccc}
+ & 0 & $a$ & $b$ & $c$ & $d$ & $e$ & $f$ & $g$ & $h$ & $i$ & $j$ & $n$ \\
\hline 0 & 0 & $a$ & $b$ & $c$ & $d$ & $e$ & $f$ & $g$ & $h$ & $i$ & $j$ & $n$ \\
$a$ & $a$ & 0 & $e$ & 1 & $h$ & $b$ & $k$ & $m$ & $d$ & $j$ & $i$ & $l$ \\
$b$ & $b$ & $e$ & 0 & $f$ & $i$ & $a$ & $c$ & $l$ & $j$ & $d$ & $h$ & $m$ \\
$c$ & $c$ & 1 & $f$ & 0 & $g$ & $k$ & $b$ & $d$ & $m$ & $l$ & $n$ & $j$ \\
$d$ & $d$ & $h$ & $i$ & $g$ & 0 & $j$ & $l$ & $c$ & $a$ & $b$ & $e$ & $k$ \\
$e$ & $e$ & $b$ & $a$ & $k$ & $j$ & 0 & 1 & $n$ & $i$ & $h$ & $d$ & $g$ \\
$f$ & $f$ & $k$ & $c$ & $b$ & $l$ & 1 & 0 & $i$ & $n$ & $g$ & $m$ & $h$ \\
$g$ & $g$ & $m$ & $l$ & $d$ & $l$ & $n$ & $i$ & 0 & 1 & $f$ & $k$ & $e$ \\
$h$ & $h$ & $d$ & $j$ & $m$ & $a$ & $i$ & $n$ & 1 & 0 & $e$ & $b$ & $f$ \\
$i$ & $i$ & $j$ & $d$ & $l$ & $b$ & $h$ & $f$ & $f$ & $e$ & 0 & $a$ & 1 \\
$j$ & $j$ & $i$ & $h$ & $n$ & $e$ & $d$ & $m$ & $k$ & $b$ & $a$ & 0 & $c$ \\
$n$ & $n$ & $l$ & $m$ & $j$ & $k$ & $g$ & $h$ & $e$ & $f$ & 1 & $c$ & 0
\end{tabular}

and

\begin{tabular}{c|cccccccccccc}
$\cdot$ & 0 & $a$ & $b$ & $c$ & $d$ & $e$ & $f$ & $g$ & $h$ & $i$ & $j$ & $n$ \\
\hline 0 & 0 & 0 & 0 & 0 & 0 & 0 & 0 & 0 & 0 & 0 & 0 & 0 \\
$a$ & 0 & $a$ & 0 & 0 & $d$ & $a$ & 0 & $d$ & $h$ & $d$ & $h$ & $h$ \\
$b$ & 0 & $b$ & 0 & 0 & $c$ & $b$ & 0 & $c$ & $f$ & $c$ & $f$ & $f$ \\
$c$ & 0 & 0 & $b$ & $c$ & 0 & $b$ & $f$ & $c$ & 0 & $b$ & $b$ & $f$ \\
$d$ & 0 & 0 & $a$ & $d$ & 0 & $a$ & $h$ & $d$ & 0 & $a$ & $a$ & $h$ \\
$e$ & 0 & $e$ & 0 & 0 & $g$ & $e$ & 0 & $g$ & $n$ & $g$ & $n$ & $n$ \\
$f$ & 0 & $b$ & $b$ & $c$ & $c$ & 0 & $f$ & 0 & $f$ & $f$ & $c$ & 0 \\
$g$ & 0 & 0 & $b$ & $g$ & 0 & $e$ & $n$ & $g$ & 0 & $e$ & $e$ & $n$ \\
$h$ & 0 & $a$ & $e$ & $g$ & 0 & $e$ & $n$ & $g$ & 0 & $h$ & $e$ & $n$ \\
$i$ & 0 & $b$ & $a$ & $d$ & $c$ & $e$ & $h$ & $g$ & $f$ & 1 & $k$ & $n$ \\
$j$ & 0 & $e$ & $a$ & $d$ & $g$ & $b$ & $h$ & $c$ & $n$ & $m$ & $l$ & $f$ \\
$n$ & 0 & $e$ & $e$ & $g$ & $g$ & 0 & $n$ & 0 & $n$ & $n$ & $g$ & 0
\end{tabular}

$\left(N S R_{1}\right),\left(N S R_{2}\right),\left(N S R_{3}\right),\left(N S R_{4}\right)$, and $\left(N S R_{5}\right)$ properties have to hold in $N_{r}(B)^{*} S$ for all elements of $S$. However, sum or multiplication of elements in $N_{r}(B)^{*} S$ may not always belong to $N_{r}(B)^{*} S($ or $\mathcal{O})$. For instance, $d+f=l \notin \mathcal{O}$ for $d, f \in N_{r}(B)^{*} S, a+c=1 \notin N_{r}(B)^{*} S$ for $a, c \in N_{r}(B)^{*} S, j \cdot j=l \notin N_{r}(B)^{*} S$ for $j \in N_{r}(B)^{*} S$.

Theorem 6. Let $S$ be a nearness semiring and $\left\{P_{i} \mid i \in I\right\}$ be a set of prime (resp. semiprime) ideals of $S$ where an arbitrary index set $I$.

i) If $N_{r}(B)^{*}\left(\bigcap_{i \in I} P_{i}\right)=\bigcap_{i \in I} N_{r}(B)^{*} P_{i}$, then $\bigcap_{i \in I} P_{i}$ is a prime (resp. semiprime) ideal of $S$.

ii) $\bigcup_{i \in I} P_{i}$ is a prime (resp. semiprime) ideal of $S$. 
Proof. i) From Theorem 3 (i), we get $\bigcap_{i \in I} P_{i}$ is an ideal of $S$. Let $A_{1} \cdot A_{2} \subseteq N_{r}(B)^{*}\left(\bigcap_{i \in I} P_{i}\right)$ for any two ideals $A_{1}$ and $A_{2}$ of $S$. Then, $A_{1} \cdot A_{2} \subseteq \bigcap_{i \in I} N_{r}(B)^{*} P_{i}$ by hypothesis, and hence $A_{1} \cdot A_{2} \subseteq N_{r}(B)^{*} P_{i}$ for all $i \in I$. Since $P_{i}$ are prime ideals of $S$ for all $i \in I, A_{1} \subseteq P_{i}$ or $A_{2} \subseteq P_{i}$ for all $i \in I$. In this case, $A_{1} \subseteq \bigcap_{i \in I} P_{i}$ or $A_{2} \subseteq \bigcap_{i \in I} P_{i}$.

ii) $\bigcup_{i \in I} P_{i}$ is an ideal of $S$ by Theorem 3 (ii). Let $A_{1} \cdot A_{2} \subseteq N_{r}(B)^{*}\left(\bigcup_{i \in I} P_{i}\right)$ for any ideals $A_{1}$ and $A_{2}$ of $S$.Then, $A_{1} \cdot A_{2} \subseteq \bigcup_{i \in I} N_{r}(B)^{*} P_{i}$ by Theorem 1.(ii). There is at least one $i_{0} \in I$ such that $A_{1} \cdot A_{2} \subseteq N_{r}(B)^{*} P_{i_{0}}$. Since $P_{i_{0}}$ are prime ideals of $S$ for $i_{0} \in I, A_{1} \subseteq P_{i_{0}}$ or $A_{2} \subseteq P_{i_{0}}$ for $i_{0} \in I$. Therefore, $A_{1} \subseteq \bigcup_{i \in I} P_{i}$ or $A_{2} \subseteq \bigcup_{i \in I} P_{i}$.

Theorem 7. Let $S$ be a nearness semiring and $a, b \in S$. If $P$ is a prime right ideal of $S$ such that $N_{r}(B)^{*}\left(N_{r}(B)^{*} P\right)=N_{r}(B)^{*} P$, then $a \cdot S \cdot b \subseteq N_{r}(B)^{*} P$ implies $a \in P$ or $b \in P$.

Proof. Let $a \cdot S \cdot b \subseteq N_{r}(B)^{*} P$. In this case, we have $(a \cdot S \cdot b) \cdot S \subseteq\left(N_{r}(B)^{*} P\right) \cdot S \subseteq$ $N_{r}(B)^{*} P$ by Theorem 2 (ii). Therefore, by Lemma $2 a \cdot S$ and $b \cdot S$ are right ideals of $S$, and since $P$ is prime right ideal of $S, a \cdot S \subseteq P$ or $b \cdot S \subseteq P$. There exists $e \in N_{r}(B)^{*} S$ such that $a=e a$ for all $a \in S$. Therefore, either $a \in P$ or $b \in P$.

We give the following theorem without the proof.

Theorem 8. Let $S$ be a nearness semiring and $a \in S$. If $P$ is a semiprime right ideal of $S$ such that $N_{r}(B)^{*}\left(N_{r}(B)^{*} P\right)=N_{r}(B)^{*} P$, then $a \cdot S \cdot a \subseteq N_{r}(B)^{*} P$ implies $a \in P$.

Theorem 9. Let $S$ be a nearness semiring, $P$ be a right ideal of $S$ such that $N_{r}(B)^{*}\left(N_{r}(B)^{*} P\right)=N_{r}(B)^{*} P$ and $a, b \in S$. If $a \cdot S \cdot b \subseteq N_{r}(B)^{*} P$ implies $a \in P$ or $b \in P$, then $P$ is a prime right ideal of $S$.

Proof. Let $A_{1}$ and $A_{2}$ be any two right ideals of $S$ such that $A_{1} \cdot A_{2} \subseteq N_{r}(B)^{*} P$ and $A_{1} \nsubseteq P$. Thus, there exists an element $a_{1} \in A_{1}$ such that $a_{1} \notin P$. For any $a_{2} \in A_{2}$, we have $a_{1} \cdot S \cdot a_{2}=\left(a_{1} \cdot S\right) \cdot a_{2} \subseteq\left(N_{r}(B)^{*} A_{1}\right) \cdot a_{2}$. On the other hand, let $x \in\left(N_{r}(B)^{*} A_{1}\right) \cdot a_{2}$ such that $x=\sum_{i=1} x_{i} a_{2} ; x_{i} \in N_{r}(B)^{*} A_{1}, a_{2} \in A_{2}, 1 \leq i \leq n$. Then $x_{i} \in N_{r}(B)^{*} A_{1} \Rightarrow\left[x_{i}\right]_{B_{r}} \cap A_{1} \neq \varnothing \Rightarrow c \in\left[x_{i}\right]_{B_{r}}, c \in A_{1} \Rightarrow x_{i} \sim_{B_{r}} c, c \in A_{1}$, $1 \leq i \leq n$. Since $\sim_{B_{r}}$ is a congruence indiscernibility relation on $S$, we get that $x_{i} a_{2} \sim{ }_{B_{r}} c a_{2}, c a_{2} \in A_{1} \cdot A_{2} \subseteq N_{r}(B)^{*} P, 1 \leq i \leq n$. Since $P$ is a right ideal of $S$ such that $N_{r}(B)^{*}\left(N_{r}(B)^{*} P\right)=N_{r}(B)^{*} P$, we get that $\sum_{i=1} x_{i} a_{2} \sim_{B_{r}} \sum c a_{2}$, $\sum c a_{2} \in N_{r}(B)^{*} P, 1 \leq i \leq n$. Hence, we have $\sum c a_{2} \in\left[\sum_{i=1} x_{i} a_{2}\right]_{B_{r}}$ and $\sum c a_{2} \in$ 
$N_{r}(B)^{*} P$. Therefore, $\left[\sum_{i=1} a_{i} b\right]_{B_{r}} \cap\left(N_{r}(B)^{*} P\right) \neq \varnothing \Rightarrow[x]_{B_{r}} \cap\left(N_{r}(B)^{*} P\right) \neq \varnothing$, so we obtain $x \in N_{r}(B)^{*}\left(N_{r}(B)^{*} P\right)=N_{r}(B)^{*} P$, namely, $a_{1} \cdot S \cdot a_{2} \subseteq N_{r}(B)^{*} P$. By hypothesis, we have $a_{2} \in P$, and so $A_{2} \subseteq P$. This completes the proof.

Theorem 10. Let $S$ be a nearness semiring, $P$ be a right ideal of $S$ such that $N_{r}(B)^{*}\left(N_{r}(B)^{*} P\right)=N_{r}(B)^{*} P$, and $a \in S$. If $a \cdot S \cdot a \subseteq N_{r}(B)^{*} P$ implies $a \in P$, then $P$ is a semiprime right ideal of $S$.

Definition 9. Let $S$ be a nearness semiring, $A_{1}, A_{2}$ and $P$ be ideals of $S . P$ is called an irreducible (resp. a strongly irreducible) ideal of $S$ if $A_{1} \cap A_{2}=N_{r}(B)^{*} P$ (resp. $\left.A_{1} \cap A_{2} \subseteq N_{r}(B)^{*} P\right)$ implies $A_{1}=P$ or $A_{2}=P\left(\right.$ resp. $A_{1} \subseteq P$ or $\left.A_{2} \subseteq P\right)$.

Theorem 11. Let $S$ be a nearness semiring and $\left\{A_{i} \mid i \in I\right\}$ be a set of ideals of $S$ where an arbitrary index set I. If $N_{r}(B)^{*}\left(\bigcap_{i \in I} A_{i}\right)=\bigcap_{i \in I} N_{r}(B)^{*} A_{i}$, then every strongly irreducible and semiprime ideal of $S$ is a prime ideal of $S$.

Proof. Let $N_{r}(B)^{*}\left(\bigcap_{i \in I} A_{i}\right)=\bigcap_{i \in I} N_{r}(B)^{*} A_{i}$ for all ideals $A_{i}$ of $S$, and $P$ be a strongly irreducible and semiprime ideal of $S$. Let $A_{1} \cdot A_{2} \subseteq N_{r}(B)^{*} P$ for any ideals $A_{1}$ and $A_{2}$ of $S$. Then, $A_{1} \cap A_{2}$ is a ideal of $S$ by Theorem 3 (i). Therefore, $\left(A_{1} \cap A_{2}\right)^{2}=\left(A_{1} \cap A_{2}\right) \cdot\left(A_{1} \cap A_{2}\right) \subseteq A_{1} \cdot A_{2} \subseteq N_{r}(B)^{*} P \Rightarrow\left(A_{1} \cap A_{2}\right)^{2} \subseteq N_{r}(B)^{*} P$. Since $P$ is a semiprime ideal of $S$, we get that $A_{1} \cap A_{2} \subseteq P$. Thus, $A_{1} \cap A_{2} \subseteq N_{r}(B)^{*} P$ by Theorem 1.(i). We get $A_{1} \subseteq P$ or $A_{2} \subseteq P$, for $P$ is a strongly irreducible ideal of $S$.

\section{Conclusion}

We have introduced the concept of prime (semiprime) ideals of semiring on weak nearness approximation spaces and we have given some properties of such ideals. One can investigate others properties of nearness semiring. Also, this paper will contribute to the application in several algebraic structures such as prime (semiprime, maximal, etc.) ideals of ring, gamma ring, gamma semiring, and etc. on weak nearness approximation spaces.

\section{REFERENCES}

[1] Biswas, R. and Nanda, S. Rough groups and rough subgroups, Bull. Pol. AC. Math, 42, (1994), 251-254.

[2] Davvaz, B. Rough sets in a fundamental ring, Bull. Iranian Math. Soc, 24(2), (1998), 49-61.

[3] Davvaz, B. Roughness in rings, Inform. Sci, 164(1-4), (2004), 147-163.

[4] Golan, J. S. Semirings and Their Applications, Kluwer Academic Publishers, 1999.

[5] İnan, E. and Öztürk, M. A. Near groups on nearness approximation spaces, Hacet. J. Math. Stat, 41(4), (2012), 545-558.

[6] İnan, E. and Öztürk, M. A. Erratum and notes for near groups on nearness approximation spaces, Hacet. J. Math. Stat, 43(2), (2014), 279-281. 
[7] İnan, E. and Öztürk, M. A. Near semigroups on nearness approximation spaces, Ann. Fuzzy Math. Inform, 10(2), (2015), 287-297.

[8] Iwinski, T. B. Algebraic approach to rough sets, Bull. Pol. AC. Math, 35, 1987, 673-683.

[9] Kuroki, N. Rough ideals in semigroups, Inform. Sci, 100(1-4), (1997), 139-163.

[10] Miao, D.; Han, S.; Li, D. and Sun, L. Rough group, rough subgroup and their properties, International Workshop on Rough Sets, Fuzzy Sets, Data Mining, and Granular-Soft Computing, Springer-Verlag, Heidelberg, 104-113, 2005.

[11] Öztürk, M. A. and İnan, E. Soft nearness approximation spaces, Fund. Inform, 124(1), (2013), $231-250$.

[12] Öztürk, M. A.; Uçkun, M. and İnan, E. Near groups of weak cosets on nearness approximation spaces, Fund. Inform, 133, (2014), 433-448.

[13] Öztürk, M. A.; Çelik Siner, İ. and Jun, Y. B. Nearness BCK-algebras, Int. J. Open Problems Compt. Math, 8(4), (2015) 37-57.

[14] Öztürk, M. A. and İnan, E. Nearness rings, Ann. Fuzzy Math. Inform 2019, (In press).

[15] Öztürk, M. A.; Jun, Y. B. and İz, A. Gamma semigroups on weak nearness approximation spaces, J. Int. Math. Virtual Inst, 9, (2019), 53-72.

[16] Öztürk, M. A. Semiring on weak nearness approximation spaces, Ann. Fuzzy Math. Inform, 15(3), (2018), 227-241.

[17] Pawlak, Z. Classification of objects by means of attributes, Institute for Computer Science, Polish Academy of Sciences, Report 429, 1981.

[18] Pawlak, Z. Rough sets, Int. J. Comput. Inform. Sci, 11(5), (1982) 341-356.

[19] Peters, J. F. Near sets: General theory about nearness of objects, Appl. Math. Sci, 1(53-56), (2007), 2609-2629.

[20] Peters, J. F. Near sets: Special theory about nearness of objects, Fund. Inform, 75(1-4), (2007), 407-433.

[21] Peters, J. F. Classification of perceptual objects by means of features, Int. J. Info. Technol. Intell. Comput, 3(2), (2008), 1-35.

[22] Peters, J. F. Near sets: An introduction, Math. Comput. Sci, 7(1), (2013), 3-9.

[23] Selvan, V. and Senthil Kumar, G. Rough ideals in semirings, Int. J. Math. Sci. Appl, 2(2), (2012), 557-564.

Current address: Department of Mathematics, Faculty of Arts and Sciences, Adiyaman University, 02040 Adiyaman, Turkey.

E-mail address: mehaliozturk@gmail.com

ORCID Address: http://orcid.org/0000-0002-1721-1053

Current address: Department of Mathematics Science Institute Adiyaman University 02040 Adiyaman, Turkey.

E-mail address: irfantemur02@gmail.com

ORCID Address: http://orcid.org/0000-0002-1846-2743 